\title{
Deep ultraviolet resonant Raman imaging of a cell
}

Yasuaki Kumamoto

Atsushi Taguchi

Nicholas Isaac Smith

Satoshi Kawata

\section{O SPIE}




\title{
Deep ultraviolet resonant Raman imaging of a cell
}

\author{
Yasuaki Kumamoto, ${ }^{a}$ Atsushi Taguchi, ${ }^{a}$ Nicholas Isaac Smith, ${ }^{b}$ and Satoshi Kawata, \\ aRIKEN, Nanophotonics Laboratory, 2-1 Hirosawa, Wako, Saitama 351-0198, Japan \\ bOsaka University, Immunology Frontier Research Center, 3-1 Yamadaoka, Suita, Osaka 565-0871, Japan \\ 'Osaka University, Department of Applied Physics, 2-1 Yamadaoka, Suita, Osaka 565-0871, Japan
}

\begin{abstract}
We report the first demonstration of deep ultraviolet (DUV) Raman imaging of a cell. Nucleotide distributions in a HeLa cell were observed without any labeling at $257 \mathrm{~nm}$ excitation with resonant bands attributable to guanine and adenine. Obtained images represent DNA localization at nucleoli in the nucleus and RNA distribution in the cytoplasm. The presented technique extends the potential of Raman microscopy as a tool to selectively probe nucleic acids in a cell with high sensitivity due to resonance. $\odot 2012$ Society of Photo-Optical Instrumentation Engineers (SPIE). [DOI: 10.1117/1.JBO.17.7.076001]
\end{abstract}

Keywords: Raman spectroscopy; ultraviolet; microscopy.

Paper 12103 received Feb. 15, 2012; revised manuscript received May 17, 2012; accepted for publication May 25, 2012; published online Jul. 3, 2012.

Raman scattering spectroscopy is a useful tool for biochemical and analytical study of a molecule of interest. The technique probes a specimen without any molecular labeling. This is because Raman scattering light has an energy shift, which is inherent to the chemical structure of a molecule, relative to the excitation light. Analyzing a spectrum derived from a molecule, we can collect the valuable information on chemical and physical characteristics of the molecule, such as molecular structure and electronic state.

When combined with microscopy, Raman spectroscopy (Raman microscopy) becomes a label-free imaging technique to reveal molecular distributions in a cell. ${ }^{1-5}$ Raman microscopy applications were reported for sugar, ${ }^{6}$ lipid, ${ }^{7-9}$ protein, ${ }^{8-12}$ metal complex, ${ }^{8,9,11}$ nucleic acid, ${ }^{5,12,13}$ and carotenoid ${ }^{13}$ molecules in cells. With the help of fast imaging techniques, such as slit confocal $^{8,9}$ and nonlinearscattering ${ }^{14,15}$ microscopy, Raman spectroscopy achieved dynamic molecular imaging of cells.

Varying the excitation wavelength, Raman microscopy probes different molecular species in a cell. Blue/green excitation light highlights visible pigments such as carotene ${ }^{13}$ and heme-complex $8,9,11$ in a cell. This is caused by the resonance Raman effect, where the scattering cross-section increases when the excitation wavelength is close to a molecular electronic transition band. Optimal resonance results in enhancement of up to $10^{6}$ compared with the nonresonant case. Even without significant resonance, at red to near-infrared wavelengths, abundant molecules in a cell are highlighted, such as $\operatorname{lipid}^{7}$ and protein. ${ }^{5,10,12}$

Away from the visible spectrum, deep ultraviolet (DUV) resonantly excites Raman scattering of nucleotide bases ${ }^{16,17}$ and amino acid residues ${ }^{18,19}$ that have strong absorption bands generated by electronic transition in DUV. Because of resonance, DUV Raman spectroscopy can sensitively probe specific structures, which are not probed by off-resonance wavelengths, and can uniquely measure microstructure and conformation of proteins $^{19}$ and nucleic acids. ${ }^{20}$ One study using DUV Raman

Address all correspondence to: Satoshi Kawata, Osaka University, Immunology Frontier Research Center, 3-1 Yamadaoka, Suita, Osaka 565-0871, Japan. Tel: +81 6879 7845; Fax: +81 6879 7876; E-mail: kawata@ap.eng.osaka-u.ac.jp spectroscopy analyzed the secondary structure of telomeric DNA to study the lifetime of living organisms. ${ }^{21}$ Another study showed secondary structure changes in myoglobin linked with the Tyr and Trp residues micro-environment. ${ }^{22}$ The method is also used for cellular level analysis: using resonance, DUV can selectively observe such aromatic compounds in a cell. ${ }^{23-25}$ Cellular conditions,${ }^{26}$ types, ${ }^{27}$ and metabolic states ${ }^{28}$ have all been studied using DUV Raman, and these aromatic compounds play important roles in dynamic activities and functions of cells.

In this study, we extended DUV Raman spectroscopy of cells to microscopy imaging, which has not been reported previously. To achieve this, we developed and optimized a conventional stage-scanning DUV laser Raman microscope for cellular imaging with sub-micron resolution while minimizing molecular photodegradation induced by DUV in order to gain sufficient signal before molecules are significantly degraded.

Figure 1(a) shows an obtained Raman spectrum of a HeLa cell together with a single spectrum derived from the substrate. Three prominent bands seen at $1340,1490,1580 \mathrm{~cm}^{-1}$ are all assigned to guanine and adenine, ${ }^{29,30}$ while a band at $1615 \mathrm{~cm}^{-1}$ is attributable to tyrosine and tryptophan. ${ }^{27}$ We chose the band at $1490 \mathrm{~cm}^{-1}$ for cellular nucleotide imaging. The distribution of the intensity at $1490 \mathrm{~cm}^{-1}$ is shown in Fig. 1(b). In the image, we see two intense circular spots situated close together near the center, indicated by arrows. Comparison with the transmission image shown in Fig. 1(c) confirms that these spots exist inside the nucleus, indicating they correspond to nucleoli containing dense DNA. The comparison also reveals that nucleotides are distributed in the cytoplasm with a high concentration. Such a distribution would be expected due to RNA existing in the cytoplasm.

In some cells, we found a clear difference in the intensity distribution of different two bands (1490 and $\left.1340 \mathrm{~cm}^{-1}\right)$. Figure 2(a) shows two spectra obtained in different locations in a HeLa cell, and the distribution of 1490 and $1340 \mathrm{~cm}^{-1}$ is shown in Fig. 2(b) and 2(c), respectively. In the $1340 \mathrm{~cm}^{-1}$ image, the nucleus is clearly highlighted. The highlighted distribution in the $1340 \mathrm{~cm}^{-1}$ image is understood to represent the

0091-3286/2012/\$25.00 (C) 2012 SPIE 

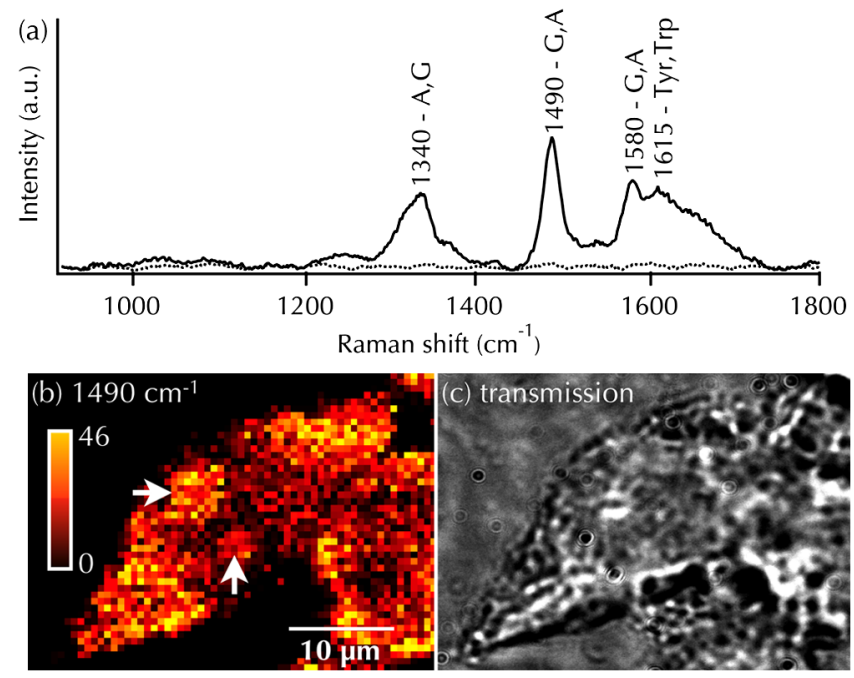

Fig. 1 DUV Raman microscopy of a HeLa cell: (a) A single spectrum of the cell (solidline) and substrate (dashed line)l (b) a image reconstructed with the intensity at $1490 \mathrm{~cm}^{-1}$; including color scale bar; and (c) transmission image corresponding to (b). The spectrum was numerically processed for the noise removal before used for the image reconstruction; briefly, acquired spectra were first processed with singular value decomposition (SVD), then hyperspectral data was reconstructed with loading vectors that contribute to the image contrast. Finally, each spectrum was processed with baseline background subtraction using polynomial function fitting. ${ }^{8}$
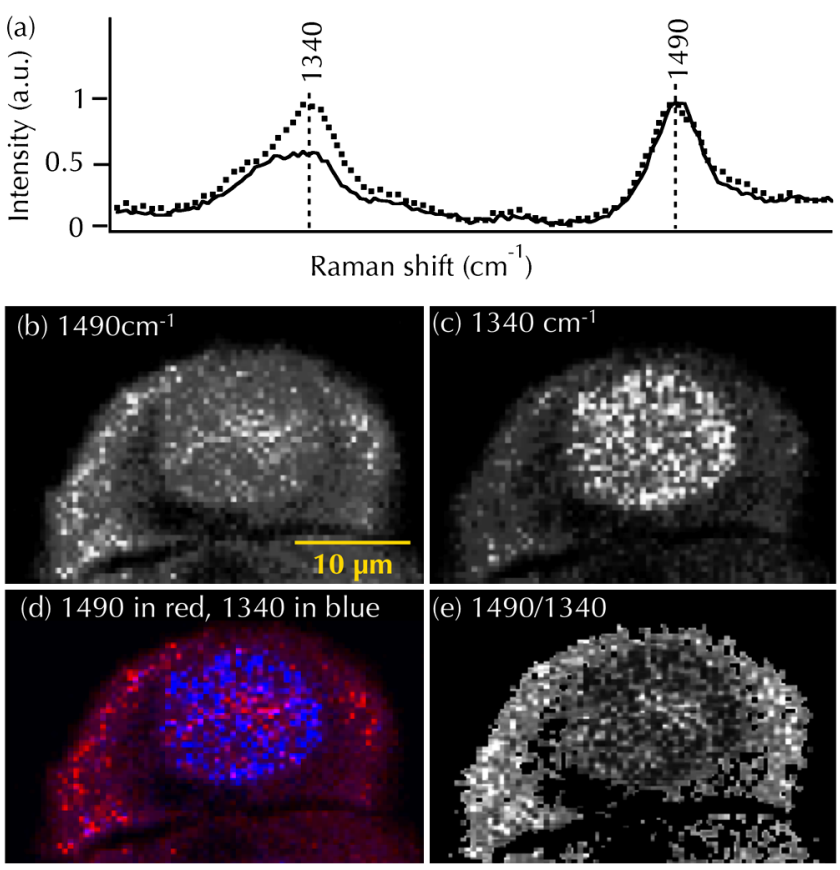

Fig. 2 (a) DUV Raman spectrum deriving from different locations in a HeLa cell: (b) and (c) Images reconstructed by intensity at (b) 1490, and (c) $1340 \mathrm{~cm}^{-1}$; (d) ahyperspectral image of two bands. The distribution shown in (b) and (c) are shown in red, and blue, respectively. (e) A ratio image (1490/1340).

DNA distribution in the nucleus. ${ }^{26}$ On the other hand, the $1490 \mathrm{~cm}^{-1}$ image highlights parts of the cytoplasm outside the nucleus. As mentioned above, the images show distribution of both DNA and RNA in a cell, and the difference between the two distributions is clearly seen in their overlapping image [see
Fig. 2(d)]. Then it is suggested that the ratio image of the $1490 \mathrm{~cm}^{-1}$ image to the $1340 \mathrm{~cm}^{-1}$ image (1490/1340) can generate a map of RNA distribution, shown in Fig. 2(e). Note that because this is a ratio image, the denominator was processed by thresholding at an appropriate noise floor before generating the ratio image. The image is not very clear, but does show a characteristic distribution of RNA in a cell, which is relatively low in the nucleus and higher in the cytoplasm. ${ }^{31}$ The results show how DUV Raman microscopy can be used for distinguishing distributions, such as DNA and RNA in a cell without any molecular labeling.

The difference in reconstructed images from 1340 and $1490 \mathrm{~cm}^{-1}$ bands may emerge from the difference in the contribution ratio of adenine and guanine to the respective bands. A report experimentally obtaining scattering cross-sections of deoxyadenosine and deoxyguanosine ${ }^{29}$ supports our idea that scattering cross-sections of adenine and guanine for the $1490 \mathrm{~cm}^{-1}$ band are close, while the band at $1340 \mathrm{~cm}^{-1}$ is dominated by adenine with a slight guanine contribution. This means that the $1340 \mathrm{~cm}^{-1}$ image highlights adenine distribution, while the $1490 \mathrm{~cm}^{-1}$ image shows adenine and guanine net distribution. To our knowledge, there is no proof that either adenine is richer, or guanine is less, in DNA compared to RNA. Further study is needed to conclude the origin of difference between two images; however, the present results and discussions imply that DUV Raman microscopy is capable of distinguishing the distribution of adenine and guanine in a cell.

As mentioned above, molecular degradation initiated by DUV (photodegradation) was a significant challenge, ${ }^{32,33}$ which has long limited the use of DUV Raman spectroscopy in microscopy. The photon energy is so high that electrons in an absorbing molecule are excited, and the molecule can irreversibly change through energy relaxation such as ionization. In addition, reactive by products generated can destroy nearby biomolecules. Additionally, performance of optical components, such as the lens and detector for DUV, has been largely limited. Thus the photodegradation constrains the acquisition of spectral signal sufficient for molecular identification from a small volume of sample before molecular damage. ${ }^{33}$ There are some studies reporting DUV Raman microspectroscopy of cells, but to get sufficient $\mathrm{S} / \mathrm{N}$, the area for spectral acquisition was relatively large. ${ }^{30,34-36}$ One report showed $3 \times 9 \mu \mathrm{m}^{2}$ pixel-based microspectroscopy of paramecium cells, ${ }^{35}$ while another study on plant samples used $5 \mu \mathrm{m}^{2}$ pixels. ${ }^{36}$ Sub-micron resolution is essential for molecular imaging of a cell.

There were essentially four main challenges overcome to achieve the present technique. The first is to optimize the intensity and exposure duration of excitation. In a previous study, we showed appropriate excitation conditions for Raman microscopy of cells by correlating intensity and exposure with photodegradation during measurement. ${ }^{33}$ The second is to improve the detection throughput of a microscope (details below). The third is to find the right scanning step of the sample stage. The step was set at $0.5 \mu \mathrm{m}$, which is almost twice as large as the Airy disk diameter at the sample plane, so that photodegradation does not accumulate during imaging. The fourth is sample preparation. The fourth is sample preparation and is related to the step size issue mentioned above. In experiments, by determining the onset of photodegradation, ${ }^{33}$ we can then operate in a range where obtained spectra are not degraded and reconstructed images show clear molecular distributions. Photodegradation limits the effective resolution of the microscope if 
the photodegradation region exceeds the focal spot. In our experiments (data not shown), the resolution for imaging desiccated cells was as small as $0.35 \mu \mathrm{m}$. On the other hand, for inliquid cells, even cells chemically fixed with paraformaldehyde, the molecular photodegradation region was wider, and therefore the pixel resolution for in-liquid cells can not be smaller than $1.2 \mu \mathrm{m}$. Those fourkeys enabled DUV Raman microscopy of a cell with submicron spatial resolution.

Design of the purpose-made microscope is an important part for this research. While similar to experimental setups for visible wavelength Raman imaging, ${ }^{2,3}$ our system has several unique features. For excitation, the microscope uses the $257 \mathrm{~nm} \mathrm{cw}$ line from a frequency-doubled $\mathrm{Ar}^{+}$laser (Coherent, Inc.), suitable for resonant excitation of nucleotide bases. ${ }^{30}$ Even with desiccated samples, we used a high NA glycerin-immersion objective lens (Carl Zeiss, Inc. NA $=1.35$ ) for excitation and scattering light collection from the sample with the smallest possible excitation focal volume. The excitation intensity is adjusted to $0.1 \mathrm{~mW} / \mu \mathrm{m}^{2}$ by an ND filter. A shutter is used for controlling the duration of sample exposure to avoid photodegradation of the sample. Rayleigh scattering is rejected by an edgefilter, and the frequency-processed light is then dispersed by a holographic grating (Princeton Instruments, Inc. $1800 \mathrm{G} / \mathrm{mm})$. We measured the spectra by a back-illuminated CCD (Princeton Instruments, Inc. SPEC-10 2KBUV). Grating dispersion is calibrated with the laser line $\left(0 \mathrm{~cm}^{-1}\right)$, and Raman bands of boron nitride $\left(1370 \mathrm{~cm}^{-1}\right)$ and acetonitrile $\left(2249 \mathrm{~cm}^{-1}\right)$. For imaging, a piezo-electric stage (PI, Inc.) raster-scanned the sample with a $0.5 \mu \mathrm{m}$ step. Each spectrum is acquired with $0.75 \mathrm{~s}$ sample exposure, and all optics are necessarily made of quartz or $\mathrm{CaF}_{2}$.

Due to signal-to-noise considerations, some noise reduction is required. We decomposed hyperspectral images by unwrapping the three-dimensional (3-D) hyperstack into a twodimensional (2-D) matrix of wavenumber and pixel dimension. We then performed singular value decomposition (SVD) and reconstructed the spectral based on only the top five loading vectors, which contribute strongly to spectral image contrast. For data shown here, use of the top five loading vectors appeared optimum. In some cases a loading vector containing useful information may fall outside the top five, so that manual inspection of loading vectors is still required to ensure that maximum spectral information is retained. Due to the poor signal-to-noise ratio of raw spectra, in some cases, using background subtraction before SVD and reconstruction produced errors. We therefore first applied SVD and reconstruction before considering background removal. The contribution of autofluorescence from tryptophan or other components will be spatially overlapped with the Raman signal, and SVD did not produce complete separation of these spectral distributions. Therefore we did an additional per-pixel polynomial function fit and subtraction to remove remaining background. The resulting images show quite uniform background (dark regions outside the cell) due to the strength of this processing at removing random noise that does not contribute to the spectral information in the data.

It is useful to further discuss spatial variation of spectra to understand how valid this imaging method is for cellular nucleotide imaging. For the image in Fig. 1, a mean spectrum from a large number of pixels in the cell region $(n=2225)$ and then in the substrate area $(n=1155)$ for pre-processed data is given in Fig. 3. Spectra are shown as mean spectra, together with plus/ minus standard deviation curves in Fig. 3(a). This shows the
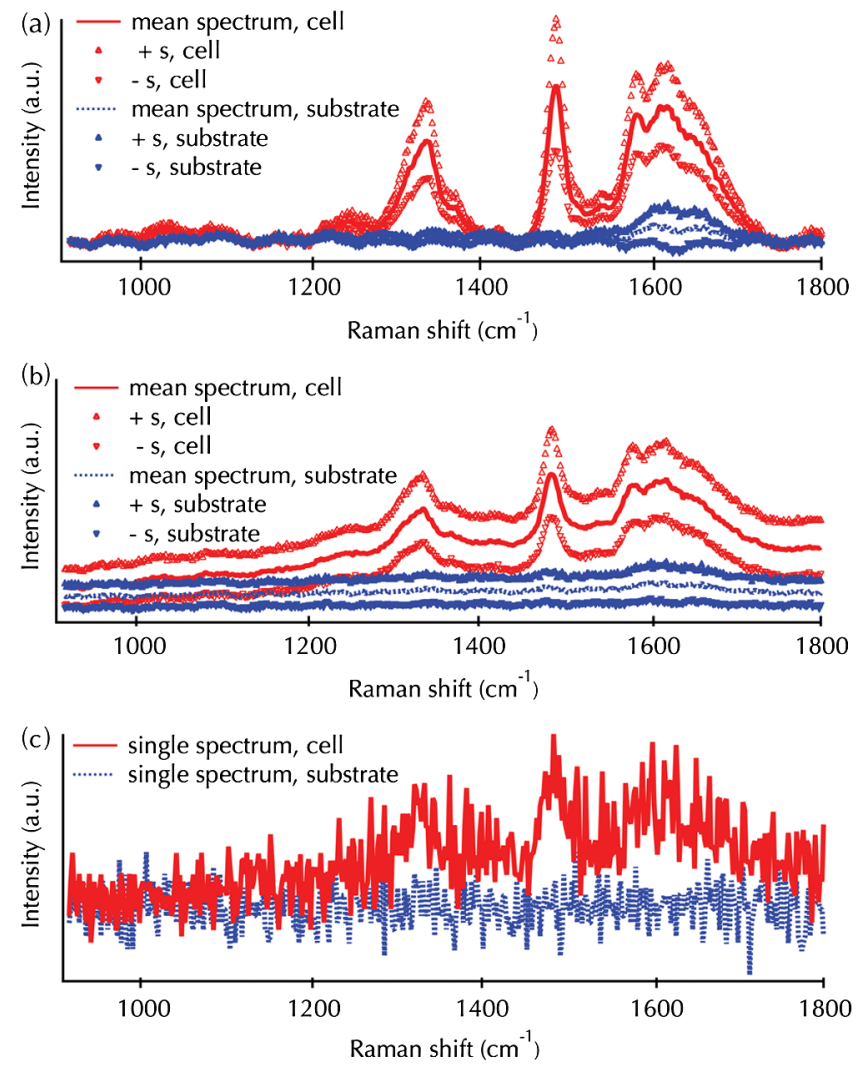

Fig. 3 Mean spectra of cell and substrate regions for the data set in Fig. 1: The top panel (a) shows pre-processed data (see main text for details); (b) shows raw data. Mean curves are shown together with curves representing plus/minus standard deviation; (c) a representative single pixel spectrum from both the cell area and the substrate region.

spectral variation reproducibility for the measurement. For both the cell and substrate, the standard deviation is low enough that the separation of cell and background is obvious, indicating good reproducibility of the method, even though by nature, pixels in the cell area must vary throughout the whole cell area. Figure 3(b) shows the same measurements, without preprocessing. Clear signals are still observable, allowing the use of raw data, although the usefulness of the processing technique becomes clear. The standard deviation is, again, small and is a similar level to the noise. This is apparent in Fig. 3(c), which shows spectrum from a single pixel. We find that the presented method should be reliable for resonant observation of aromatic component distributions in cells.

In conclusion, we demonstrated the first DUV Raman microscopy imaging of a cell. Using $257 \mathrm{~nm}$ excitation, we selectively obtained nucleotide distribution in a HeLa cell, by utilizing the resonant Raman effect. Obtained cell spectral signals from pixels within the cell are well-separated from spectra due to the substrate and are reproducible, making further spectral analysis possible. The presented technique extends the potential of Raman microscopy as a tool to probe nucleic acids in a cell with high sensitivity. Future improvement of the signal detection throughput will further develop the technique as a useful label-free tool to analyze nucleic acid dynamics during biological processes such as apoptosis and mitosis.

At the end, we note the overall picture of the DUV research field. DUV optics and light sources have been specifically developed and revamped in the past decade. Because of this, DUV 
studies for microscopy ${ }^{31,37}$ lithography, sterilization, and other fields are growing. Recently, plasmonics, with the features of electric field enhancement and spatial confinement, is also budding in DUV and preliminary applications for enhanced Raman scattering were reported. ${ }^{38-40}$ DUV research field has a huge potential for future applications.

\section{Acknowledgments}

This study was supported by RIKEN research funding and by JSPS via Grant-in-aid for scientific research $S$ program. The authors thank Dr. Katsumasa Fujita (Osaka university) for valuable comments and discussions.

\section{References}

1. G. J. Puppels et al., "Studying single living cells and chromosomes by confocal Raman microspectroscopy," Nature 347(6290), 301-303 (1990).

2. G. J. Puppels, M. Grond, and J. Greve, "Direct imaging Raman microscope based on tunable wavelength excitation and narrow-band emission detection," Appl. Spectrosc. 47(8), 1256-1267 (1993).

3. N. M. Sijtsema et al., "Confocal direct imaging Raman microscope: design and applications in biology," Appl. Spectrosc. 52(3), 348-355 (1998).

4. Y.-S. Huang et al., "Molecular-level investigation of the structure, transformation, and bioactivity of single living fission yeast cells by time- and space-resolved Raman spectroscopy," Biochemistry 44(30), 10009-10019 (2005).

5. N. Uzunbajakava et al., "Nonresonant confocal Raman imaging of DNA and protein distribution in apoptotic cells," Biophys. J. 84(6), 3968-3981 (2003).

6. F. C. Pascut et al., "Toward label-free Raman-activated cell sorting of cardiomyocytes derived from human embryonic stem cells," J. Biomed. Opt. 16(4), 045002 (2011).

7. H.-J. van Manen et al., "Single-cell Raman and fluorescence microscopy reveal the association of lipid bodies with phagosomes in leukocytes," Proc. Natl. Acad. Sci. 102(29), 10159-10164 (2005).

8. K. Hamada et al., "Raman microscopy for dynamic molecular imaging of living cells," J. Biomed. Opt. 13(4), 044027 (2008).

9. M. Okada et al., "Label-free Raman observation of cytochrome c dynamics during apoptosis," Proc. Natl. Acad. Sci. 109(24), 28-32 (2012).

10. N. Uzunbajakava et al., "Nonresonant Raman imaging of protein distribution in single human cells," Biopolymers 72(1), 1-9 (2003).

11. H.-J. van Manen et al., "Intracellular chemical imaging of hemecontaining enzymes involved in innate immunity using resonance Raman microscopy," J. Phys. Chem. B 108(48), 18762-18771 (2004).

12. A. B. Zoladek et al., "Label-free molecular imaging of immunological synapses between dendritic and $T$ cells by Raman micro-spectroscopy," Analyst 135(12), 3205-3212 (2010).

13. V. V. Pully, A. T. M. Lenferink, and C. Otto, "Time-lapse Raman imaging of single live lymphocytes," J. Raman Spectrosc. 42(2), 167-173 (2011).

14. C. L. Evans et al., "Chemical imaging of tissue in vivo with video-rate coherent anti-Stokes Raman scattering microscopy," Proc. Natl. Acad. Sci. 102(46), 16807-16812 (2005).

15. B. G. Saar et al., "Video-rate molecular imaging in vivo with stimulated Raman scattering," Science 330(6009), 1368-1370 (2010).

16. M. Pezolet, T.-J. Yu, and W. L. Peticolas, "Resonance and preresonance Raman spectra of nucleotides using ultraviolet lasers," J. Raman Spectrosc. 3(1), 55-64 (1975).

17. S. R. A. Fodor et al., "Ultraviolet resonance Raman spectroscopy of the nucleotides with 266-, 240-, 218-, and $200 \mathrm{~nm}$ pulsed laser excitation," J. Am. Chem. Soc. 107(6), 1520-1529 (1985).
18. A. Y. Hirakawa et al., "Characterization of a few Raman lines of tryptophan," J. Raman Spectrosc. 7(5), 282-287 (1978).

19. C. R. Johnson et al., "UV resonance Raman spectroscopy of the aromatic amino acids and myoglobin," J. Am. Chem. Soc. 106(17), 5008-5010 (1984).

20. S. R. A. Fodor and T. G. Spiro, "Ultraviolet resonance Raman spectroscopy of DNA with 200-266 nm laser excitation," J. Am. Chem. Soc. 108(12), 3198-3205 (1986).

21. C. Krafft, J. M. Benevides, and G. J. Thomas Jr., "Secondary structure polymorphism in Oxytricha nova telomeric DNA," Nucl. Acids Res. 30(18), 3981-3991 (2002).

22. Z. Chi and S. A. Asher, "UV resonance Raman determination of protein acid denaturation: selective unfolding of helical segments of horse myoglobin," Biochemistry 37(9), 2865-2872 (1998).

23. K. A. Britton et al., "Ultraviolet resonance Raman spectra of Escherichia coli with 222.5-251.0 nm pulsed laser excitation," Appl. Spectrosc. 42(5), 782-788 (1988).

24. S. Nocentini and L. Chinsky, "In vivo studies of nucleic acid by ultraviolet resonance Raman spectroscopy on eucaryotic living cells," J. Raman Spectrosc. 14(1), 9-10 (1983).

25. R. A. Dalterio et al., "An ultraviolet ( $242 \mathrm{~nm}$ excitation) resonance Raman study of live bacteria and bacterial components," Appl. Spectrosc. 41(3), 417-422 (1987).

26. Y. Yazdi et al., "Resonance Raman spectroscopy at $257 \mathrm{~nm}$ excitation of normal and malignant cultured breast and cervical cells," Appl. Spectrosc. 53(1), 82-85 (1999).

27. Q. Wu et al., "UV Raman spectral intensities of E. coli and other bacteria excited at 228.9, 244.0, and $248.2 \mathrm{~nm}, "$ Anal. Chem. 73(14), 3432-3440 (2001).

28. U. Neugebauer et al., "Towards a detailed understanding of bacterial metabolism-spectroscopic characterization of Staphylococcus epidermidis," ChemPhysChem 8(1), 124-137 (2007).

29. Z. Q. Wen and G. J. Thomas Jr., "UV resonance Raman spectroscopy of DNA and protein constituents of viruses: assignments and cross sections for excitations at 257, 244, 238, 229 nm," Biopolymers 45(3), 247-256 (1998).

30. S. Chadra, W. H. Nelson, and J. F. Perry, "Ultraviolet micro-Raman spectrograph for the detection of small numbers of bacterial cells," Rev. Sci. Instrum. 64(11), 3088-3093 (1993).

31. M. C. Cheung et al., "Deep ultraviolet mapping of intracellular protein and nucleic acid in femtograms per pixel," Cytom. Part A 79A(11), 920-932 (2011).

32. M. Harz et al., "UV-resonance Raman spectroscopic study of human plasma of health donors and patients with thrombotic microangiopathy," Biopolymers 82(4), 317-324 (2006).

33. Y. Kumamoto et al., "Deep UV resonant Raman spectroscopy for photodamage characterization in cells," Biomed. Opt. Express 2(4), 927-936 (2011).

34. F. Sureau et al., "An ultraviolet micro-Raman spectrometer: resonance Raman spectroscopy within single living cells," Appl. Spectrosc. 44(6), 1047-1051 (1990).

35. V. Pajcini et al., "UV Raman microspectroscopy: spectral and spatial selectivity with sensitivity and simplicity," Appl. Spectrosc. 51(1), 81-86 (1997).

36. T. Frosch, M. Schmitt, and J. Popp, "In situ UV resonance Raman micro-spectroscopic localization of the antimalarial quinine in cinchona bark," J. Phys. Chem. B 111(16), 4171-4177 (2007).

37. B. J. Zeskind et al., "Nucleic acid and protein mass mapping by live-cell deep-ultraviolet microscopy," Nat. Methods 4(7), 567-569 (2007).

38. T. Doerfer, M. Schmitt, and J. Popp, "Deep-UV surface-enhanced Raman scattering," J. Raman Spectrosc. 38(11), 1379-1382 (2007).

39. A. Taguchi et al., "Deep-UV tip-enhanced Raman scattering," J. Raman Spectrosc. 40(9), 1324-1330 (2009).

40. S. K. Jha et al., "Deep-UV surface-enhanced resonance Raman scattering of adenine on aluminum nanoparticle arrays," J. Am. Chem. Soc. 134(4), 1966-1969 (2012). 